\title{
INDICADORES DA ACIDEZ DO SOLO PARA RECOMENDAÇÃO DE CALAGEM NO SISTEMA PLANTIO DIRETO( ${ }^{(1)}$
}

\author{
Margarete Nicolodi ${ }^{(2)}$, Ibanor Anghinoni ${ }^{(3)} \&$ Clesio Gianello $^{(4)}$
}

\begin{abstract}
RESUMO
O tempo de cultivo do solo no sistema plantio direto (SPD) altera a magnitude das relações entre os componentes da acidez do solo e o rendimento das culturas. Altos rendimentos podem ser obtidos nesse sistema mesmo em áreas com alta acidez. Assim, é provável que o critério de calagem utilizado no sistema convencional (SC) de cultivo não seja adequado para o SPD. O objetivo deste trabalho foi avaliar os critérios para recomendação de calagem em seis lavouras no SPD representativas da região do Planalto do RS. Foram selecionadas lavouras com alta variabilidade dos indicadores de acidez do solo. Para cada lavoura, foram avaliados 20 locais. Em cada local, foram coletadas amostras de solo das camadas de 0-10 e 0-20 cm e foi avaliado o rendimento de grãos. Nas amostras de solo, foram determinados o pH em água e $\mathrm{CaCl}_{2}$, o índice SMP, os teores de $\mathrm{Ca}, \mathrm{Mg}$ e $\mathrm{Al}$ trocáveis, de MO, de $\mathrm{P}$ e $\mathrm{K}$ disponíveis. Com base nas relações entre os indicadores de acidez do solo e o rendimento de grãos, concluiu-se que nenhum dos indicadores de acidez do solo, quando utilizados isoladamente, descreve de modo adequado o rendimento das plantas no solo cultivado no SPD consolidado. Nas duas camadas avaliadas, o rendimento relativo foi sempre maior que $65 \%$ quando o $\mathrm{pH}$ em água e em $\mathrm{CaCl}_{2}$ foi maior que 5,5 e 5,1, respectivamente; a saturação por bases, maior que $65 \%$; o Al trocável, menor que $0,3 \mathrm{cmol}_{\mathrm{c}} \mathrm{dm}^{-3}$; e a saturação por $\mathrm{Al}$, menor que $5 \%$. Para avaliação da acidez do solo em SPD com a finalidade de recomendar calagem, podem ser utilizadas tanto a camada de 0-10 como a de 0-20 cm de profundidade.
\end{abstract}

Termos de indexação: pH, saturação por alumínio e por bases, rendimento de grãos.

\footnotetext{
(1) Parte da Dissertação de Mestrado da primeira autora apresentada à Universidade Federal do Rio Grande do Sul - UFRGS. Trabalho realizado, em parte, com apoio financeiro do CNPq e PRONEX. Recebido para publicação em maio de 2006 e aprovado em outubro de 2007.

(2) Pós-Doutoranda do Programa de Pós-Graduação em Ciência do Solo da Universidade Federal do Rio Grande do Sul - UFRGS. Av. Bento Gonçalves 7712, Caixa Postal 15100, CEP 90001-970 Porto Alegre (RS). E-mail: mnicolodi@ibest.com.br

(3) Professor do Programa de Pós-Graduação em Ciência do Solo, UFRGS. E-mail: ibanghi@ufrgs.br

(4) Professor do Programa de Pós-Graduação em Ciência do Solo, UFRGS. E-mail: gianello@portoweb.com.br.
} 


\title{
SUMMARY: SOIL ACIDITY INDICATORS FOR LIMING IN NO-TILLAGE SYSTEMS
}

\begin{abstract}
No-tillage systems can change the ratio between soil acidity components and crop yields over the course of time. High crop yields are not seldom observed in highly acid soils. The liming criteria established for conventional tillage soils are likely to be unsuitable for no-tillage soils. The objective of this report was to define liming criteria, based on the yield response to different soil acidity levels and layers in no-tillage systems. Six plantations were sampled in the Planalto region of the state of Rio Grande do Sul, Brazil, with very distinct soil acidity conditions, and that have been cultivated in no-tillage system over a long period. Soil samples were taken from two soil layers $(0-10$ and $0-20 \mathrm{~cm})$ and yields determined at 20 sites of each plantation. Based on regressions between relative grain yield and soil acidity indexes, it can be concluded that none of the soil acidity indicators individually can properly describe crop yield in the no-tillage system. Though significant, the relations between soil acidity indexes and crop yield were low and similar, independent of the soil layer. Thus, either soil layer can be used for liming diagnostic in no-tillage.
\end{abstract}

Index terms: $p H$, aluminum saturation, base saturation, no-tillage, grain yield.

\section{INTRODUÇÃO}

A maioria das culturas responde à calagem em solos muito ácidos, como os que predominam nas regiões tropicais e subtropicais. Essa é uma prática que favorece o desenvolvimento do sistema radicular, assegura o aproveitamento dos nutrientes e aumenta a rentabilidade econômica da agricultura nesse tipo de solo. No entanto, os maiores benefícios da calagem são obtidos quando esta é associada à aplicação adequada de fertilizantes e às demais práticas agrícolas recomendadas (Ribeiro et al., 1999).

Na elaboração das recomendações para calagem, são definidos os critérios de calagem, os métodos para determinação da quantidade de corretivo para corrigir a acidez numa camada de solo e o modo de aplicação dos corretivos de acidez em função das culturas da região, do sistema de preparo e cultivo e do tipo de solo. Os principais indicadores de acidez utilizados para recomendação de calagem no Brasil são: o pH em água, no Rio Grande do Sul e em Santa Catarina (RS/SC); a saturação por bases, em São Paulo e em alguns Estados vizinhos; o teor de alumínio (Al) trocável, nos Estados do Nordeste, Norte e parte do Centro-Oeste; e o teor de $\mathrm{Al}$, cálcio (Ca) e magnésio $(\mathrm{Mg})$ trocáveis, na região de Cerrado. Os métodos para determinação da quantidade de corretivo são, respectivamente, o índice SMP, a saturação por bases da CTC a pH 7,0, a neutralização do Al trocável e o teor de Ca e de Mg trocáveis do solo (CFS RS/SC, 1995; Raij et al., 1996; Ribeiro et al., 1999; Wiethölter, 2000; Sousa \& Lobato, 2004).

As recomendações para calagem foram definidas, nas diferentes regiões do Brasil, para os sistemas de cultivo que mobilizam o solo, como o sistema convencional (SC), em que o corretivo de acidez é incorporado na camada arável, cuja dinâmica é conhecida. A avaliação da necessidade de calagem e a correção da acidez do solo neste sistema são feitas na camada de 0-20 cm, e a necessidade de nova aplicação, reavaliada após cinco anos. Em geral, as recomendações de calagem na implantação do sistema plantio direto (SPD) são as mesmas do SC; no entanto, quando o SPD está em fase consolidada, normalmente após cinco anos da adoção, os efeitos da mudança do sistema de cultivo no solo são evidentes, com melhor desenvolvimento das plantas. Alguns benefícios do cultivo no SPD em relação ao SC são: o aumento dos teores de matéria orgânica (MO) e de nutrientes, a diminuição da toxidez do $\mathrm{Al}$ às plantas, a melhoria da estrutura e da capacidade de retenção de água do solo, o aumento da fertilidade na camada superficial do solo e a preservação das relações construídas com o tempo de cultivo, principalmente pelo não-revolvimento e conseqüente manutenção dos resíduos das culturas na superfície $(0-10 \mathrm{~cm})$ (Salet, 1998; Sá, 1999; Mielniczuk et al., 2000; Anghinoni \& Nicolodi, 2004).

O funcionamento do sistema solo depende das condições iniciais, da magnitude e da continuidade dos fluxos de matéria e de energia e da interação entre seus subsistemas químico, físico e biológico e destes com os sistemas vegetal, atmosférico e antrópico (Nicolodi et al., 2004b). Quanto maior o tempo de cultivo no SPD, maior a complexidade das estruturas formadas no sistema solo, o qual se auto-organiza em nível de ordem mais alto e gera propriedades emergentes que podem beneficiar o seu funcionamento e o desenvolvimento das plantas (Vezzani, 2001). No SC, em geral, ocorre o inverso: diminuem os teores de MO, a infiltração e a capacidade de retenção de água e aumentam a erosão e o empobrecimento do solo (Mielniczuk et al., 2000). Desse modo, é provável que, no solo cultivado por longo período no SPD, as interações (MO x Al trocável, MO x $\mathrm{P}$ disponível, estrutura $\mathrm{x}$ retenção de água, etc.) se manifestem mais intensamente, influenciando a acidez, a disponibilidade e a absorção de nutrientes e o rendimento das culturas 
(Nicolodi et al., 2004a). Diversos trabalhos (Anghinoni \& Salet, 1998; Caires et al., 1998; Pöttker \& Ben, 1998; Sá, 1999; Gianello \& Nicolodi, 2004) indicam a menor influência da acidez na produtividade das culturas no SPD consolidado (mais de cinco anos neste sistema), pois, freqüentemente, têm sido observados rendimentos elevados em muitas áreas com alta acidez do solo. Isso indica que o critério (indicador de acidez e seu valor de referência) utilizado para recomendar calagem no SC pode não ser adequado para solos cultivados no SPD. Além disso, muitos dos benefícios do tempo de cultivo do solo neste sistema se manifestam na forma de gradientes no perfil do solo, sendo necessário adequar a espessura da camada avaliada e a correção de acidez com a camada da maior absorção de nutrientes pelas plantas (Anghinoni \& Salet, 1998).

A toxidez por Al é o fator que mais limita a produção das culturas em solos ácidos. Apesar de não existir um indicador estreitamente associado à fitotoxicidade do $\mathrm{Al}$ no solo, o $\mathrm{pH}$ é utilizado universalmente para diagnosticar o estado de acidez do solo e indicar a necessidade ou não de calagem (Kaminski, 1989). No entanto, a relação entre o $\mathrm{pH}$ e a toxidez por $\mathrm{Al}$ e, ou, Mn depende do tipo de solo, do teor e do tipo de MO, do nível de fertilidade, do ambiente e da época do ano (Sumner, 1997). O aumento dos teores de MO no SPD determina também um aumento da complexação do $\mathrm{Al}$ trocável e, dependendo do tipo de ligante envolvido no processo e do tipo do complexo formado, é possível a presença de $\mathrm{Al}$ trocável com valor de $\mathrm{pH}$ baixo, sem causar toxidez às plantas (Salet, 1998). Por isso, o pH em água, isoladamente, pode não ser um indicador eficiente da toxidez por $\mathrm{Al}$ e, conseqüentemente, para recomendação de calagem nesse sistema.

A definição do critério de calagem requer a existência de uma rede experimental regional, com culturas (espécies de plantas) representativas da produção de grãos e solos com ampla faixa de acidez. A utilização de lavouras no SPD com ampla variação nos indicadores de acidez pode também ser uma opção vantajosa ao uso de áreas experimentais, pela maior representatividade dos dados, pois as recomendações são elaboradas para essas condições. Considerando as mudanças promovidas no sistema solo com o tempo de cultivo no SPD, é importante estudar qual o tipo de acidez do solo que mais limita o rendimento das culturas neste sistema consolidado. Os objetivos deste trabalho foram selecionar os indicadores de acidez do solo em uma determinada camada amostrada e estabelecer seus valores de referência para recomendação de calagem no SPD em lavouras da região do Planalto do RS.

\section{MATERIAL E MÉTODOS}

Para o trabalho, foram selecionadas seis lavouras, cujo critério de seleção foi o de terem alta variabilidade nos indicadores de acidez (teor de Al trocável, saturação por $\mathrm{Al}$ e por bases e $\mathrm{pH}$ ) para bem representar as condições para as quais são elaboradas as recomendações de calagem. As lavouras estão localizadas em três municípios da região do Planalto do Rio Grande do Sul e, por ocasião da avaliação, já estavam no SPD há pelo menos cinco anos (consolidado). A área das lavouras variou de 15 a 20 ha em Latossolo Vermelho distrófico. As lavouras 1, 2 e 3 localizam-se no município de Cruz Alta; a lavoura 4, no município de Não-Me-Toque; e as lavouras 5 e 6 , no município de Ibirubá, sendo cinco cultivadas com soja (Glycine max Merril) e uma com cevada (Hordeum vulgare) e posteriormente com soja. Os tratos culturais e a adubação foram feitos conforme as Indicações Técnicas da Soja RS/SC (2001) e as Indicações Técnicas para Produção de Cevada Cervejeira (Comissão..., 2001), com adubação na linha de semeadura e adubação de cobertura a lanço. Embora as culturas tenham sido manejadas de modo semelhante, o histórico de uso das áreas era diferente (Quadros 1 e 2). Em Cruz Alta, a expansão das lavouras, em substituição à pecuária tradicional sobre campo natural, é relativamente recente, especialmente a partir da década de 1990, enquanto em Ibirubá e em Não-Me-Toque a agricultura é praticada desde a década de 1960 no SC e, a partir do início da década de 1990, no SPD.

Foram avaliados 20 locais $(2,0 \times 2,4 \mathrm{~m})$ em cada lavoura, distribuídos de modo a contemplar a heterogeneidade do solo. Em cada local, foi coletada uma amostra de solo, composta por três subamostras, nas camadas de 0-10 e 0-20 cm de profundidade, e avaliado o rendimento de grãos. Cada subamostra de solo foi coletada com pá-de-corte e consistiu de uma fatia de $5 \mathrm{~cm}$ de espessura por $40 \mathrm{~cm}$ de largura, antes do cultivo da soja, e de $20 \mathrm{~cm}$, antes do cultivo da cevada, centralizada na linha de semeadura. Todas as plantas de cada local foram cortadas, manualmente, no final do ciclo das culturas (cevada, safra $2001 \mathrm{e}$ soja, safra 2001/02), para avaliação do rendimento de grãos. Estas plantas foram trilhadas, os grãos pesados e a sua umidade corrigida para $13 \%$, para cálculo do rendimento por hectare. Nas amostras de solo, foram determinados o $\mathrm{pH}$ em água; o índice SMP; os teores de $\mathrm{Ca}$, de $\mathrm{Mg}$ e de $\mathrm{Al}$ trocáveis, de $\mathrm{MO}$, de $\mathrm{K}$ e de $\mathrm{P}$ disponíveis (Mehlich-1), conforme Tedesco et al. (1995); o pH em $\mathrm{CaCl}_{2} 10 \mathrm{mmol} \mathrm{L}^{-1}$, conforme Silva (1999); o valor da acidez potencial $(\mathrm{H}+\mathrm{Al})$ foi estimado pelo índice SMP, utilizando-se as equações de Escosteguy \& Bissani (1999) e de Kaminski et al. (2001); e a saturação por bases e por $\mathrm{Al}$, por cálculo.

$\mathrm{Na}$ avaliação dos resultados, foi calculado o rendimento relativo de grãos para cada cultura, atribuindo-se o valor 100 ao rendimento máximo em cada lavoura avaliada, e os demais valores foram proporcionais a este. Foram estabelecidas relações entre o rendimento relativo de grãos e os indicadores de acidez do solo, para definição do critério de calagem. 
Quadro 1. Histórico de cultivo da safra de verão 1998/99 e de inverno de 2001 das lavouras conduzidas no sistema plantio direto na região do Planalto do RS, avaliadas nesse trabalho

\begin{tabular}{|c|c|c|c|c|c|c|}
\hline \multirow{2}{*}{ Lavouras } & \multicolumn{3}{|c|}{ Verão 1998/99 } & \multicolumn{3}{|c|}{ Inverno 1999} \\
\hline & Cultura & Adubação(1) & Rend. grãos & Cultura & Adubação ${ }^{1}$ & Rend.grãos \\
\hline & & $\mathrm{kg} \mathrm{ha}^{-1}$ (fórmula) & $\mathrm{tha}^{-1}$ & & $\mathrm{~kg} \mathrm{ha}^{-1}$ (fórmula) & $\mathrm{tha} \mathrm{h}^{-1}$ \\
\hline 1. Cruz Alta & soja & $250(02-20-30)$ & $1,62^{(2)}$ & aveia & 200 P natural & - \\
\hline 2. Cruz Alta & milho & $200(00-20-30)$ & $2,05^{(2)}$ & trigo & $\begin{array}{l}250(08-18-28) \\
+200 \text { uréia }\end{array}$ & 1,64 \\
\hline 3. Cruz Alta & milho & $175(02-20-20)$ & $1,56^{(2)}$ & aveia + azevém & - & - \\
\hline 4. Não-Me-Toque & milho & $\begin{array}{l}300(05-25-25) \\
+100 \text { uréia }\end{array}$ & 5,10 & cevada & $250(05-25-25)$ & 2,28 \\
\hline 5. Ibirubá & soja & $200(02-20-30)$ & 2,70 & trigo & $200(05-20-30)$ & 1,98 \\
\hline \multirow[t]{2}{*}{ 6. Ibirubá } & soja & $200(02-20-30)$ & 2,70 & trigo & $200(05-20-30)$ & 2,10 \\
\hline & \multicolumn{3}{|c|}{ Verão 1999/2000 } & \multicolumn{3}{|c|}{ Inverno 2000} \\
\hline 1. Cruz Alta & soja & $250(02-20-30)$ & $1,80^{(2)}$ & trigo & $200(05-20-30)$ & 2,10 \\
\hline 2. Cruz Alta & soja & $200(00-20-30)$ & $1,68^{(2)}$ & aveia + azevém & - & - \\
\hline 3. Cruz Alta & soja & $100 \mathrm{KCl}$ & $1,50^{(2)}$ & aveia + azevém & - & - \\
\hline 4. Não-Me-Toque & soja & $200(00-20-30)$ & $2,40^{(2)}$ & trigo & $230(05-25-25)$ & 2,10 \\
\hline 5. Ibirubá & soja & $200(02-20-30)$ & $2,10^{(2)}$ & cevada & $250(05-20-30)$ & 1,20 \\
\hline \multirow[t]{2}{*}{ 6. Ibirubá } & soja & $200(02-20-30)$ & $2,88^{(2)}$ & trigo & $200(05-20-30)$ & 1,80 \\
\hline & \multicolumn{3}{|c|}{ Verão $2000 / 2001$} & \multicolumn{3}{|c|}{ Inverno 2001} \\
\hline 1. Cruz Alta & soja & $250(02-20-30)$ & 3,12 & trigo & $200(05-20-30)$ & 1,80 \\
\hline 2. Cruz Alta & soja & $200(00-20-30)$ & 2,52 & aveia + azevém & - & - \\
\hline 3. Cruz Alta & soja & $200(00-20-30)$ & 1,98 & aveia + azevém & - & - \\
\hline 4. Não-Me-Toque & soja & $200(00-20-30)$ & 3,48 & trigo & $230(08-18-28)$ & 2,88 \\
\hline 5. Ibirubá & soja & $220(02-20-30)$ & 3,30 & trigo & $200(05-20-30)$ & 1,80 \\
\hline 6. Ibirubá & soja & $190(02-20-30)$ & 3,18 & cevada & $200(05-20-30)$ & 1,92 \\
\hline
\end{tabular}

${ }^{(1)} \mathrm{N}-\mathrm{P}_{2} \mathrm{O}_{5}-\mathrm{K}_{2} \mathrm{O} .{ }^{(2)}$ Prejudicado por precipitação pluvial abaixo da normal.

Quadro 2. Tempo de cultivo do solo no SPD, amplitude e média do rendimento de grãos de soja (safra 2001/ 02) e última adubação e calagem nas lavouras conduzidas no sistema plantio direto na região do Planalto do RS, avaliadas neste trabalho

\begin{tabular}{|c|c|c|c|c|c|}
\hline \multirow[b]{2}{*}{ Lavouras } & \multirow{2}{*}{$\begin{array}{l}\text { Tempo } \\
\text { de SPD }\end{array}$} & \multicolumn{2}{|c|}{ Rendimento de grãos ${ }^{(1)}$} & \multirow{2}{*}{$\begin{array}{c}\text { Dose } \\
\text { Adubação } \\
\left(\mathrm{N}-\mathrm{P}_{2} \mathrm{O}_{5}-\mathrm{K}_{2} \mathrm{O}\right)\end{array}$} & \multirow{2}{*}{$\begin{array}{c}\text { Última aplicação } \\
\text { de calcário }\end{array}$} \\
\hline & & Amplitude & Média & & \\
\hline & Anos & \multicolumn{2}{|c|}{$\mathrm{t} \mathrm{ha}^{-1}$} & $\mathrm{~kg} \mathrm{ha}{ }^{-1}$ & Ano/t ha ${ }^{-1} / \operatorname{modo}$ \\
\hline 1. Cruz Alta & 5 & $1,8-6,0$ & 2,9 & $280(02-20-30)$ & 1998/4,0/incorporado \\
\hline 2. Cruz Alta & 9 & $2,7-4,2$ & 2,5 & $250(00-20-30)$ & $1998 / 2,7 /$ superficial \\
\hline 3. Cruz Alta & 8 & $0,3-3,4$ & 2,1 & $280(00-12-30)$ & 1993/4,0/incorporado \\
\hline 4. Não-Me-Toque & 6 & $2,3-4,4$ & 3,0 & $200(00-20-30)$ & 1995/3,0/incorporado \\
\hline 5. Ibirubá & 5 & $2,8-4,2$ & 3,1 & $200(00-20-30)$ & $2000 / 2,5 /$ superficial \\
\hline 6. Ibirubá(1) & 9 & $3,5-4,8$ & 4,0 & $200(00-20-30)$ & 1999/2,3,0/superficial \\
\hline
\end{tabular}

(1) Amplitude de rendimento de grãos de cevada 2,1-3,0 t ha ${ }^{-1}$ (safra de inverno 2001). 


\section{RESULTADOS E DISCUSSÃO}

Os solos, dentro de cada lavoura e no conjunto delas, apresentaram amplitude de acidez adequada para atender ao objetivo do trabalho, que foi o de definir o critério (indicador de acidez e seu valorreferência) para recomendação da calagem nas lavouras no SPD, com alta representatividade das condições encontradas na região produtora de grãos do Rio Grande do Sul. Os valores dos indicadores de acidez, nas lavouras cultivadas de cinco a nove anos no SPD, na camada do solo de $0-20 \mathrm{~cm}$ de profundidade (Quadro 3), variaram de 4,3 a 6,5 para o pH em água, de 19 a $88 \%$ para a saturação por bases e de 0,2 a $50 \%$ para a saturação por Al. A disponibilidade de $\mathrm{P}$ no solo se distribuiu nas faixas "muito baixo", "baixo", "médio", "suficiente" e "alto", e a de K, nas faixas "médio", "suficiente" e "alto" (CFS RS/SC, 1995).

$\mathrm{O}$ rendimento de grãos de soja variou de 0,3 a 6,0 t ha-1, considerando todos os locais, e de 2,1 a 4,0, considerando a média de cada lavoura. Embora o solo de todas as lavouras avaliadas tenha sido classificado como Latossolo Vermelho distrófico típico (tipo de solo predominante na região do Planalto do RS), o rendimento de grãos de soja foi menor nas lavouras localizadas no município de Cruz Alta (Quadro 2), com solo mais ácido e textura média (36 a $42 \%$ de argila), do que nas lavouras dos municípios de Ibirubá e NãoMe-Toque, com solo, atualmente, menos ácido e textura argilosa (58 a $75 \%$ de argila).

Para descrever a relação entre o rendimento relativo de grãos e os indicadores de acidez do solo, foi utilizado o modelo sigmóide (Figuras 1e 2). Em geral, nas lavouras, a dispersão dos pontos nas curvas de resposta das culturas foi maior do que a obtida em experimentos, em que se alteram apenas um ou dois fatores, normalmente doses ou níveis de nutrientes ou de acidez. Os valores dos coeficientes de determinação $\left(R^{2}\right)$ entre o rendimento relativo de grãos e os indicadores de acidez do solo, que variaram de 0,49 a 0,57 , na camada de $0-20$, e de 0,43 a 0,53 , na camada de $0-10 \mathrm{~cm}$ (Figuras 1 e 2), foram altamente significativos $(p<0,01)$. Esses valores, embora baixos, são aceitáveis para estudos que contemplam a variabilidade natural de lavouras e comparáveis aos obtidos em experimentos de campo com grande número de observações (Sumner, 1997). Nessa situação, as plantas respondem à interação entre os componentes dos subsistemas do sistema solo, e a recomendação de calagem não deveria ser feita em função de um único indicador de acidez, como, por exemplo, o pH do solo, conforme sugerido por Raij \& Quaggio (1997). Além disso, deve-se também considerar que, muitas vezes, as alterações no solo são qualitativas e nem sempre podem ser verificadas pelos métodos tradicionais de avaliação da fertilidade do solo.

No Rio Grande do Sul e em Santa Catarina, a recomendação de calagem foi desenvolvida no SC e utiliza o valor 6,0 de $\mathrm{pH}$ do solo em água na camada de $0-20 \mathrm{~cm}$ como critério de calagem (CFS RS/SC, 1995). No entanto, no presente trabalho com lavouras no SPD, observa-se, pela tendência central dos pontos, que não ocorre aumento do rendimento de grãos com $\mathrm{pH}$ em água maior que 5,3 (Figura 1a) e com $\mathrm{pH}$ em $\mathrm{CaCl}_{2}$ maior que 5,0 (Figura $1 \mathrm{~b}$ ). Por essa interpretação, o rendimento relativo de grãos na faixa entre 65 e $100 \%$ deve ter sido afetado por outros fatores que não o $\mathrm{pH}$ do solo. Essa dispersão de pontos ocorre também com o Al trocável menor que $0,3 \mathrm{cmol}_{\mathrm{c}} \mathrm{dm}^{-3}$ (Figura 1c), com a saturação por $\mathrm{Al}$ menor que $5 \%$

Quadro 3. Amplitude dos indicadores do solo avaliados nas camadas de 0-20 e 0-10 cm de profundidade nas seis lavouras cultivadas no sistema plantio direto consolidado na região do Planalto do RS selecionadas para o trabalho

\begin{tabular}{|c|c|c|c|c|c|c|c|c|c|c|c|c|}
\hline \multirow[b]{2}{*}{ Camada de solo } & \multirow{2}{*}{$\begin{array}{l}\text { Argila } \\
0-20 \mathrm{~cm}\end{array}$} & \multicolumn{2}{|c|}{ pH em água } & \multicolumn{2}{|c|}{ Al trocável } & \multicolumn{2}{|c|}{$\begin{array}{c}\text { Saturação } \\
\text { por Al }\end{array}$} & \multicolumn{2}{|c|}{$\begin{array}{c}\text { Saturação } \\
\text { por bases }\end{array}$} & \multirow{2}{*}{\multicolumn{2}{|c|}{$\begin{array}{l}\text { P K } \\
\text { Mehlich-1 } \\
\end{array}$}} & \multirow{2}{*}{ MO } \\
\hline & & $0-20 \mathrm{~cm}$ & $0-10 \mathrm{~cm}$ & $0-20 \mathrm{~cm}$ & $0-10 \mathrm{~cm}$ & $0-20 \mathrm{~cm}$ & $0-10 \mathrm{~cm}$ & $0-20 \mathrm{~cm}$ & $0-10 \mathrm{~cm}$ & & & \\
\hline \multicolumn{4}{|c|}{$\%$} & \multicolumn{2}{|c|}{$-\mathrm{mmol}_{\mathrm{c}} \mathrm{dm}^{-3}-$} & \multicolumn{2}{|c|}{$\%$} & \multicolumn{2}{|c|}{ - } & \multicolumn{2}{|c|}{$\mathrm{mg} \mathrm{dm}^{-3}$} & $\mathrm{~g} \mathrm{~kg}^{-1}$ \\
\hline 1. Cruz Alta & $34-46$ & $4,4-5,8$ & $4,4-5,4$ & $1-17$ & $1-11$ & $1,2-50$ & $1,0-39$ & $19-72$ & $24-75$ & $5,1-30,4$ & $67-204$ & $2,0-3,4$ \\
\hline 2. Cruz Alta & $34-35$ & $4,8-5,7$ & $4,8-5,6$ & $1-8$ & $1-11$ & $0,8-16$ & $1,0-24$ & $38-68$ & $40-68$ & $3,0-15,6$ & $50-176$ & $2,1-2,9$ \\
\hline 3. Cruz Alta & $40-44$ & $4,3-5,2$ & $4,3-5,2$ & $1-22$ & $2-20$ & $1,9-45$ & $2,0-45$ & $20-64$ & $19-63$ & $2,3-28,0$ & $13-156$ & $2,4-2,7$ \\
\hline 4. Não-Me-Toque & $70-75$ & $5,0-6,2$ & $5,0-6,2$ & $0-4$ & $0-3$ & $0,3-4$ & $0,2-3$ & $54-80$ & $57-81$ & $6,7-36,6$ & $35-290$ & $3,6-3,9$ \\
\hline 5. Ibirubá & $58-65$ & $4,9-6,2$ & $5,1-6,2$ & $0-9$ & $0-6$ & $0,4-12$ & $0,1-1,9$ & $47-82$ & $62-85$ & $8,2-32,3$ & $65-158$ & $2,5-3,4$ \\
\hline 6. Ibirubá & $62-64$ & $4,7-6,5$ & $4,9-6,6$ & $0-7$ & $0-2$ & $0,2-14$ & $0,0-1,3$ & $43-88$ & $50-91$ & $3,2-25,7$ & $55-231$ & $3,3-3,8$ \\
\hline
\end{tabular}



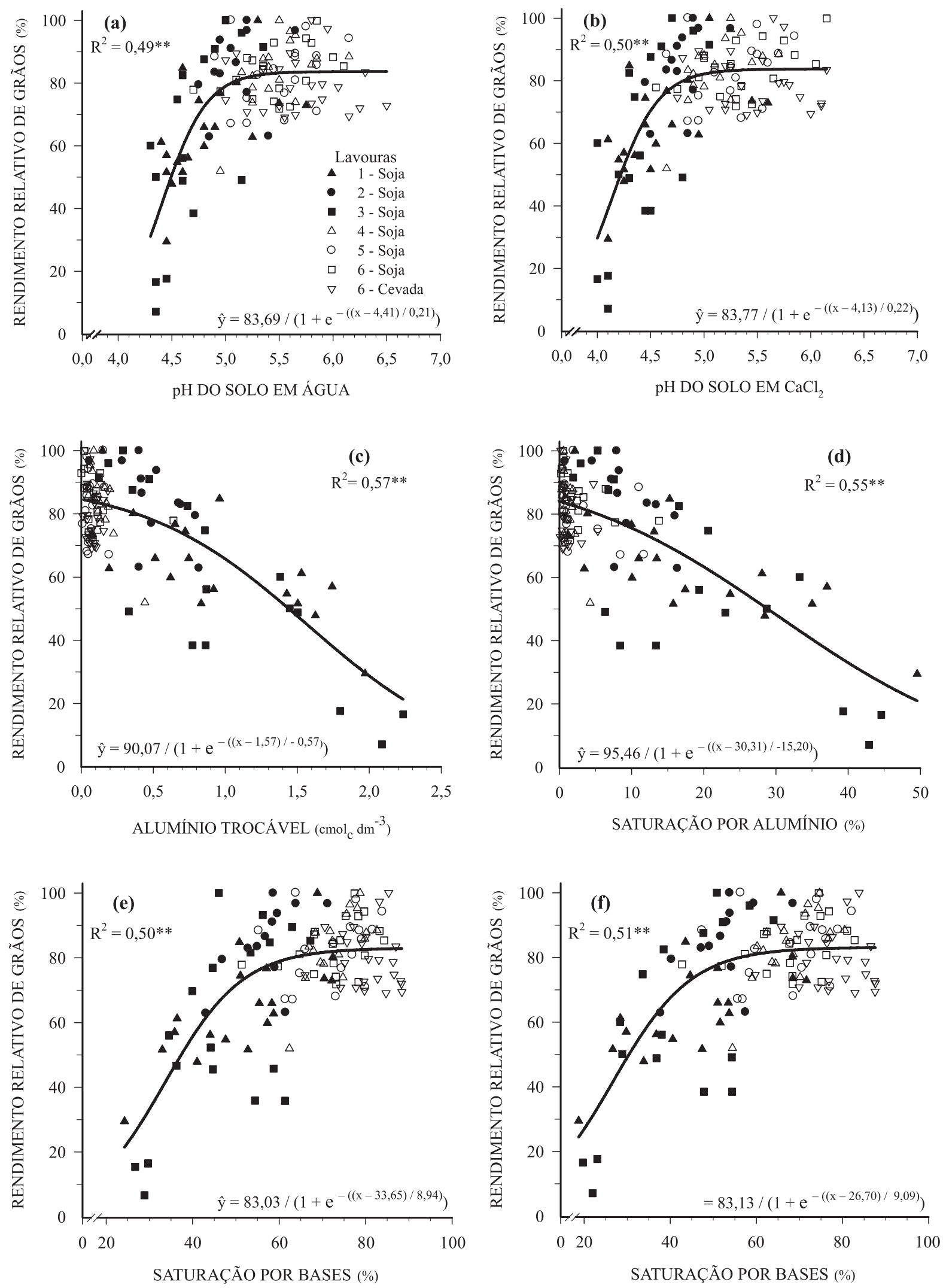

Figura 1. Relação entre o rendimento relativo de grãos e os indicadores de acidez do solo na camada de 0 a $20 \mathrm{~cm}$ de profundidade em lavouras no sistema plantio direto: (a) $\mathrm{pH}$ em água; (b) $\mathrm{pH} \mathrm{em} \mathrm{CaCl}_{2} ; \mathrm{c}$ ) alumínio trocável; (d) saturação por alumínio e saturação por bases; (e) Eq. Escosteguy \& Bissani (1999) e (f) Eq. Kaminski et al. (2001). 

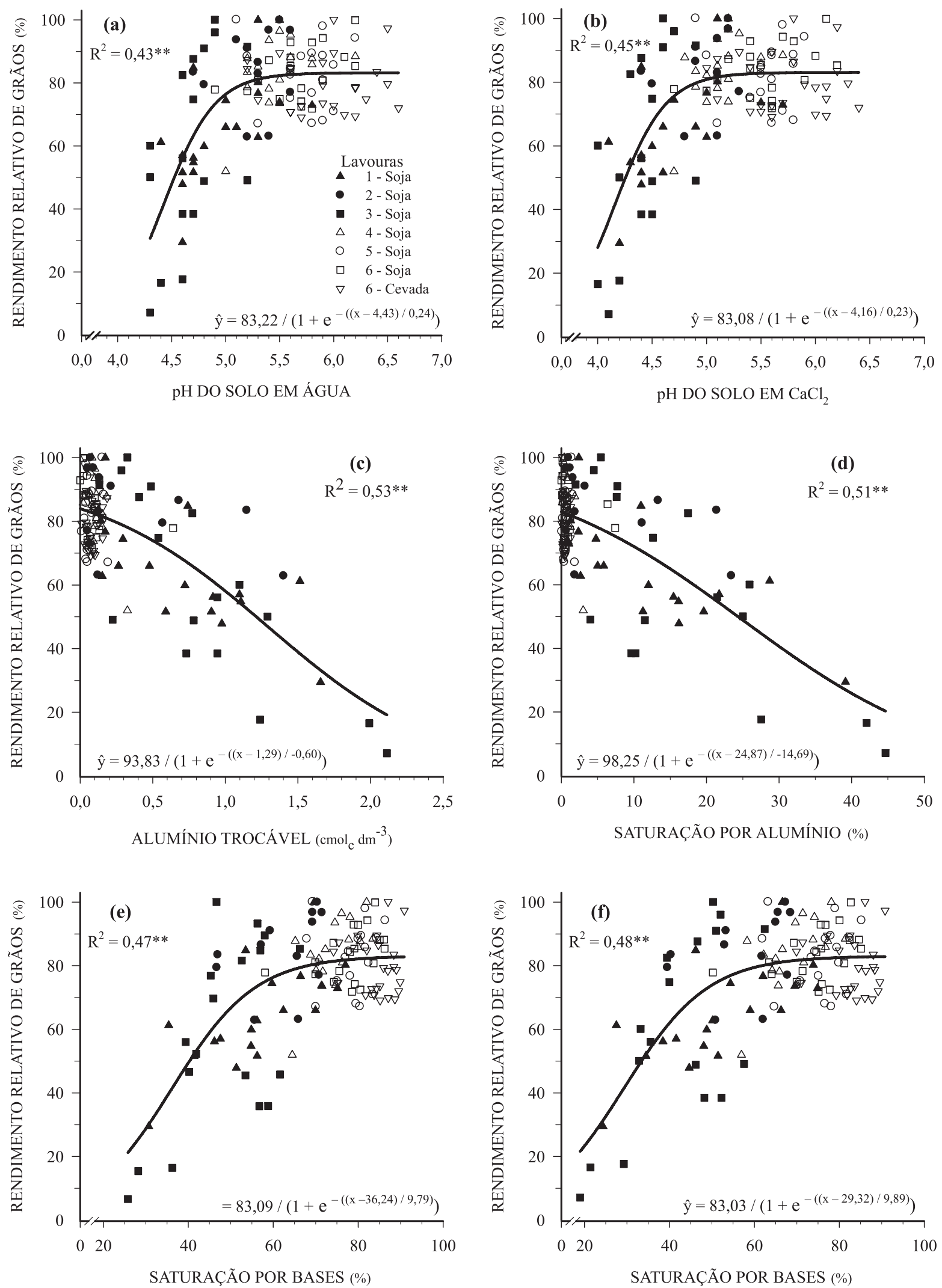

Figura 2. Relação entre o rendimento relativo de grãos e os indicadores de acidez do solo na camada de 0 a $10 \mathrm{~cm}$ de profundidade em lavouras no sistema plantio direto: (a) pH em água; (b) $\mathrm{pH} \mathrm{em}^{\mathrm{CaCl}}{ }_{2}$; (c) alumínio trocável; (d) saturação por alumínio e saturação por bases; (e) Eq. Escosteguy \& Bissani (1999) e (f) Eq. Kaminski et al. (2001). 
(Figura 1d) e com a saturação por bases maior que 60 \% (Figura 1f). Por isso, para recomendação de calagem no SPD é necessário, provavelmente, mais do que a mera avaliação química de algum fator isolado.

O grau de ajuste dos dados é maior $\left(\mathrm{R}^{2}=0,57\right)$ para relação entre o rendimento relativo de grãos e o $\mathrm{Al}$ trocável (Figura 1c) do que para o $\mathrm{pH}$ do solo, em água $\left(\mathrm{R}^{2}=0,49\right)$ e em $\mathrm{CaCl}_{2}\left(\mathrm{R}^{2}=0,50\right)$ (Figura 1a,b). Isso indica que o rendimento de grãos está mais bem relacionado com teores de $\mathrm{Al}$ trocável (acidez trocável) do que com o pH do solo (acidez ativa). Nesse caso, para maior segurança nas recomendações de calagem, é necessário manter o teor de $\mathrm{Al}$ trocável do solo menor que $0,3 \mathrm{cmol}_{\mathrm{c}} \mathrm{dm}^{-3}$, quando a avaliação é feita na camada de $0-20 \mathrm{~cm}$.

A relação entre o rendimento relativo de grãos e a saturação por $\mathrm{Al}$ (Figura 1d) é semelhante $\left(\mathrm{R}^{2}=0,55\right)$, porém um pouco menor do que o observado na relação com os teores de $\mathrm{Al}$ trocável $\left(\mathrm{R}^{2}=0,57\right)$, ambos com maiores coeficientes de determinação do que os obtidos nas relações com o $\mathrm{pH}$ do solo em água $\left(\mathrm{R}^{2}=0,49\right)$ e em $\mathrm{CaCl}_{2}\left(\mathrm{R}^{2}=0,50\right)$. Nessas lavouras, o rendimento de grãos não foi limitado pela saturação por $\mathrm{Al}$ menor que $5 \%$ (Figura 1d). O valor da saturação por $\mathrm{Al}$ de $5 \%$ é, no entanto, menor do que o proposto por Anghinoni \& Salet (2000). Esses autores propuseram o valor de $10 \%$ como limite para haver efeito do $\mathrm{Al}$ trocável no rendimento das culturas. No entanto, no presente trabalho, quando a saturação por $\mathrm{Al}$ é menor do que $5 \%$, o rendimento pode estar sendo limitado por outros fatores e não pela acidez do solo. Raij \& Quaggio (1997) verificaram, em cinco ensaios no Estado de São Paulo e um em Minas Gerais, que o rendimento das culturas de milho, de soja e de algodão em solo cultivado no SC foi muito influenciado pela alta saturação por $\mathrm{Al}(\geq 20 \%)$, e o rendimento máximo foi obtido quando a saturação foi menor que $3 \%$.

O rendimento de grãos também não foi limitado pela saturação por bases, calculada pela equação de Kaminski et al. (2001), menor do que $60 \%$, avaliada na camada de $0-20 \mathrm{~cm}$ (Figura 1f). O coeficiente de determinação da saturação por bases $\left(\mathrm{R}^{2}=0,51\right)$ é semelhante aos obtidos com o pH do solo (Figura 1a,b), porém menor que os obtidos nas relações com o Al trocável $\left(\mathrm{R}^{2}=0,57\right)$ e com a saturação por $\mathrm{Al}$ $\left(R^{2}=0,55\right)$ (Figura 1c,d).

Caires et al. (2000) concluíram que os indicadores de fertilidade da camada de $0-5 \mathrm{~cm}$ foram os que melhor se correlacionaram com o rendimento de culturas (soja, milho, trigo e triticale) em rotação por cinco anos. Por ser, no SPD, a camada mais sujeita a mudanças, a camada de $0-5 \mathrm{~cm}$, segundo os autores citados, é indicada para diagnóstico da acidez nesse sistema. Eles também observaram o efeito da calagem superficial até $10 \mathrm{~cm}$, após 12 meses de sua aplicação. No entanto, no Rio Grande do Sul, conforme dados de Anghinoni \& Salet (2000), houve efeito da calagem superficial até a profundidade de $10 \mathrm{~cm}$ em aproximadamente 36 meses após aplicação de calcário.
Os efeitos do tempo de cultivo no SPD ocorrem inicialmente na camada superficial do solo, com a formação de gradientes, principalmente de MO, de nutrientes e de acidez. Assim, é provável que a camada de $0-10 \mathrm{~cm}$ possa ser utilizada, com vantagens em relação à de $0-20 \mathrm{~cm}$, para o diagnóstico da acidez de solo na fase consolidada desse sistema. No entanto, neste trabalho, verificou-se (Figura 2) que as relações entre o rendimento relativo de grãos e todos os indicadores de acidez do solo, estes avaliados na camada de 0-10 cm, são semelhantes e têm a mesma significância $(p<0,01)$, embora, numericamente, os coeficientes sejam um pouco menores, quando comparados aos da camada de 0-20 cm (Figura 1). Da mesma forma, os valores de referência dos indicadores da acidez, em que não ocorre mais resposta em rendimento das culturas testadas (critério de calagem), são semelhantes aos obtidos na camada de 0-20 cm (Quadro 4). Algumas diferenças são verificadas somente para os valores de $\mathrm{pH}$ em água: 5,3 e 5,5, e para os de saturação por bases: 60 e $65 \%$, nas camadas $0-20$ e $0-10 \mathrm{~cm}$, respectivamente. $\mathrm{O}$ ranqueamento dos indicadores de acidez com base em valores de $\mathrm{R}^{2}$ das relações com o rendimento de grãos avaliado na camada de $0-10 \mathrm{~cm}$ foi muito semelhante ao observado na de $0-20 \mathrm{~cm}$. Verificam-se maiores valores de $\mathrm{R}^{2}$ das relações com o $\mathrm{Al}$ trocável, seguido pela saturação por $\mathrm{Al}$, pela saturação por bases e, por último, pelo $\mathrm{pH}$ em água (Figuras $1 \mathrm{e} 2$ ). Fica evidente pelos dados que, para o caso dessas lavouras, a amostragem de solos para fins de avaliação de acidez, mesmo no SPD, pode ser feita indiferentemente nas camadas de $0-10$ ou $0-20 \mathrm{~cm}$.

Os resultados deste trabalho, quando comparados aos de Nolla (2003), obtidos em colunas não-deformadas de solo em dois experimentos no mesmo tipo de solo com ampla faixa de acidez, no SPD consolidado, são diferentes quanto aos valores de referência para alguns indicadores de acidez. Por exemplo, no trabalho de

Quadro 4. Valores de referência dos indicadores da acidez do solo para recomendação de calagem para as culturas de grãos em lavouras no sistema plantio direto consolidado

\begin{tabular}{lcc}
\hline \multirow{2}{*}{$\begin{array}{c}\text { Indicador de acidez } \\
\text { do solo }\end{array}$} & $\begin{array}{c}\text { Valor de referência/ } \\
\text { camada de solo }\end{array}$ \\
\cline { 2 - 3 } & $\mathbf{0 - 2 0} \mathbf{~ c m ~}$ & $\mathbf{0 - 1 0} \mathbf{~ c m}$ \\
\hline pH do solo em água & 5,3 & 5,5 \\
pH do solo em $\mathrm{CaCl}_{2}$ & 5,0 & 5,2 \\
Alumínio trocável, cmol $\mathrm{dm}^{-3}$ & 0,3 & 0,3 \\
Saturação por alumínio, \% & 5 & 5 \\
Saturação por bases, \% & 60 & 65 \\
\hline
\end{tabular}


Nolla (2003), o valor de referência da saturação por Al passou de $17 \%$ na camada de $0-15 \mathrm{~cm}$ para $3 \%$ na camada de $0-10 \mathrm{~cm}$. Na camada de $0-10 \mathrm{~cm}$, esse autor obteve valores de referência de $50 \%$ para a saturação por bases e 4,6 para o $\mathrm{pH}$ em $\mathrm{CaCl}_{2}$, menores que os resultados obtidos neste trabalho (com dados obtidos em lavouras): de $65 \%$ e 5,2 , respectivamente. Na mesma camada de solo $(0-10 \mathrm{~cm})$, neste trabalho, o valor de referência para o $\mathrm{pH}$ em água foi 5,5 . Esses valores de referência são muito semelhantes aos observados em diversos trabalhos de pesquisa recente no SPD (Fiorin et al., 1998; Pöttker \& Ben, 1998; Gianello \& Nicolodi, 2004) e mais baixos do que os adotados pela Comissão de Fertilidade do Solo para recomendação de calagem no SPD em 1995 (CFS RS/ SC, 1995), porém idênticos aos adotados pela Comissão de Química e Fertilidade do Solo em 2004 (CQFS RS/ $\mathrm{SC}, 2004)$. No entanto, também foram obtidos bons rendimentos nestas lavouras (Figuras 1a e 2a), com valores de $\mathrm{pH}$ em água entre 5,0 e 5,5. Nas lavouras do município de Cruz Alta, os rendimentos máximos $\left(3,5,6,0\right.$ e $\left.4,2 \mathrm{tha}^{-1}\right)$ de soja foram obtidos com $\mathrm{pH}$ em água de 4,9, 5,3 e 5,5, respectivamente.

O valor de referência de $65 \%$ para a saturação por bases, na camada de $0-10 \mathrm{~cm}$, foi obtido com a CTC a $\mathrm{pH}$ 7,0 calculada com a acidez potencial $(\mathrm{H}+\mathrm{Al})$ estimada pelo índice SMP, pela equação proposta por Kaminski et al. (2001), atualmente adotada pela Rede Oficial dos Laboratórios de Análises de Solos (ROLAS RS/SC). No entanto, se no seu cálculo for utilizada a acidez potencial estimada com a equação obtida por Escosteguy \& Bissani (1999), anteriormente utilizada pela ROLAS, o seu valor de referência passa a ser de $75 \%$ (Figura 2e). Diferença semelhante (13\%) foi obtida por Nolla (2003), atribuída à subestimação da acidez potencial pela equação de Escosteguy \& Bissani (1999), conforme observado por Kaminski et al. (2001).

$\mathrm{O} \mathrm{Al} \mathrm{e} \mathrm{o} \mathrm{H}$ trocáveis são os principais componentes da acidez do solo que limitam o rendimento das culturas. Por ter alta relação com o $\mathrm{pH}$, este é utilizado para recomendação de calagem (CFS RS/SC, 1995). Por essa recomendação, há necessidade de calagem em solos cujo valor de $\mathrm{pH}$ é menor do que 6,0. Assim, em solos com $\mathrm{pH} \geq 6,0$ não se observa Al trocável. No entanto, em solos no SPD com $\mathrm{pH}$ baixo, o teor de $\mathrm{Al}$ trocável pode indicar concentração de $\mathrm{Al}$ em solução não tóxica, mesmo se presente em teores mais elevados do que os indicados como prejudiciais, dependendo do teor de MO, do tipo de ligante presente nesta e do complexo formado entre este e o $\mathrm{Al}$ (Salet, 1998). É provável, portanto, que o $\mathrm{pH}$ do solo nesse sistema limite o desenvolvimento das culturas em valores mais baixos do que o observado no SC, permitindo rendimentos altos em solos com $\mathrm{pH}$ em água menor do que 6,0 ou até menor do que 5,0 (Gianello \& Nicolodi, 2004). Isso também foi observado neste trabalho, em que os maiores rendimentos (entre 3,0 e $6,0 \mathrm{t} \mathrm{ha}^{-1}$ de soja) foram obtidos com valores de $\mathrm{pH}$ entre 4,9 e 5,8 e com valores de $\mathrm{Al}$ trocável entre 0 e $0,4 \mathrm{cmol}_{\mathrm{c}} \mathrm{dm}^{-3}$. Muitos resultados indicam que os benefícios de propriedades do solo melhoradas pelo SPD atenuam o efeito do baixo $\mathrm{pH}$ (Nicolodi et al., 2004a).

Os resultados deste trabalho confirmam que a saturação por bases pode ser um indicador de calagem tanto quanto o $\mathrm{pH}$ em água ou em $\mathrm{CaCl}_{2}$ (Figuras $1 \mathrm{e}$ $2 \mathrm{a}, \mathrm{b}, \mathrm{f})$, porém tem a vantagem de possuir fundamentação teórica mais completa (Raij \& Quaggio, 1997). Os resultados obtidos nessas lavouras também mostram que o $\mathrm{Al}$ trocável continua sendo um fator importante na definição do rendimento das culturas no SPD, mesmo considerando que os mecanismos responsáveis por sua complexação, em geral, são mais efetivos nesse sistema. Os rendimentos máximos foram sempre obtidos com teor de $\mathrm{Al}$ trocável menor que $0,5 \mathrm{cmol}_{\mathrm{c}} \mathrm{dm}^{-3}$ ou com saturação por $\mathrm{Al}$ menor que $10 \%$. Verificou-se também que nenhum rendimento foi menor que $60 \%$ do máximo com teor de $\mathrm{Al}$ trocável menor que $0,3 \mathrm{cmol}_{\mathrm{c}} \mathrm{dm}^{-3}$ ou saturação por $\mathrm{Al}$ menor que $5 \%$. No entanto, foram obtidos vários resultados maiores que $60 \%$ com valores de Al trocável entre 0,5 e $1,0 \mathrm{cmol}_{\mathrm{c}} \mathrm{dm}^{-3}$ ou saturação por $\mathrm{Al}$ entre 10 e $20 \%$.

A dispersão dos pontos na faixa de 65 a $100 \%$ de rendimento das culturas, no presente trabalho, é um indicativo de que ele foi influenciado por outros fatores. Por exemplo, na lavoura 1 observa-se que o rendimento máximo (6,0 t ha ${ }^{-1}$ - Quadro 2) foi obtido com valor de $\mathrm{pH}$ em água e $\mathrm{CaCl}_{2}$ de 5,3 e 5,1 , respectivamente, saturação por bases de $67 \%$, teor de $\mathrm{Al}$ trocável de $0,2 \mathrm{cmol}_{\mathrm{c}} \mathrm{dm}^{-3}$ e saturação por Al de $2,5 \%$ na camada de $0-10 \mathrm{~cm}$. No entanto, para a mesma lavoura e mesma camada avaliada, com o mesmo histórico (Quadros 1 e 2) de manejo, foi obtido o rendimento relativo de $73 \%$ (4,6 t ha-1 - Quadro 2$)$ com valor de $\mathrm{pH}$ em água e $\mathrm{CaCl}_{2}$ de 5,8 e 5,7, respectivamente, saturação por bases de $75 \%$, teor de $\mathrm{Al}$ trocável de $0,1 \mathrm{cmol}_{\mathrm{c}} \mathrm{dm}^{-3}$ e saturação por $\mathrm{Al}$ de $1 \%$. Considerando que as principais alterações no funcionamento do sistema solo ocorrem a partir de sua superfície no SPD, seria mais conveniente utilizar a camada de $0-10 \mathrm{~cm}$ e um conjunto de indicadores para recomendação de calagem nesse sistema. Contudo, os dados não mostram diferença entre a utilização da camada de $0-10$ ou de $0-20 \mathrm{~cm}$ de profundidade. Por outro lado, pelos resultados obtidos neste trabalho, deveriam fazer parte do conjunto de indicadores determinante da necessidade ou não de calagem a saturação por $\mathrm{Al}$, a saturação por bases e o pH. Os valores de referência sugeridos para avaliação da camada de $0-10 \mathrm{~cm}$ são de $5 \%$ para a saturação por $\mathrm{Al}, 65 \%$ para a saturação por bases e 5,5 para o $\mathrm{pH}$ em água. Neste trabalho, com o uso conjunto desses indicadores e dos seus valores de referência, critério de calagem, foi obtido um rendimento relativo de grãos mínimo superior a $60 \%$ do máximo. Na maioria dos casos, para um pH 5,5 não ocorrerá a presença de $\mathrm{Al}$ trocável será baixa; conseqüentemente, a saturação por Al será baixa e a saturação por bases será de pelo menos $65 \%$ no SPD. Por outro lado, também é possível 
que em pH abaixo de 5,5 ocorra saturação por bases acima de $65 \%$.

A alteração na magnitude das relações entre os indicadores de acidez e a fertilidade do solo no SPD foi observada em outros trabalhos (Caires et al., 1998; Salet, 1998; Sá, 1999; Pöttker \& Ben, 2000; Conceição, 2002; Anghinoni \& Nicolodi, 2004; Nicolodi et al., 2004b; Lopes et al., 2004). Para Sá (1999) e Lopes et al. (2004), nesse sistema ocorrem outros mecanismos que determinam esse comportamento, tão ou mais importantes que a diminuição da acidez do solo e que, muitas vezes, não são identificados, mesmo utilizando as avaliações químicas rotineiramente efetuadas com o intuito de conhecer a fertilidade do solo. Isso pode indicar que o atual modo de avaliar não é suficiente para entender o funcionamento do sistema solo cultivado há vários anos no SPD e, por conseguinte, para interpretar e conhecer sua fertilidade. Por isso, a revisão do conceito de fertilidade e sua avaliação no SPD são importantes, uma vez que neste sistema a fertilidade pode ser mais bem entendida como uma propriedade emergente da auto-organização do sistema solo (Nicolodi et al., 2004b). Assim, a recomendação de calagem no SPD deve priorizar o uso de indicadores do todo e considerar também as informações qualitativas para contemplar os benefícios do tempo de cultivo do solo nesse sistema.

\section{CONCLUSÕES}

1. As relações entre os indicadores de acidez e o rendimento relativo das culturas foram semelhantes e altamente significativas, independentemente da camada de solo avaliada.

2. Nenhum dos indicadores de acidez do solo, quando utilizados isoladamente, descreveu, de forma adequada, o rendimento das culturas no SPD nas lavouras avaliadas.

3. A recomendação de calagem pode ser feita com base em amostras de solos coletadas da camada de 0 $10 \mathrm{~cm}$ ou de $0-20 \mathrm{~cm}$ de profundidade, porém deve-se utilizar um conjunto de indicadores de acidez, a fim de contemplar o aumento da complexidade das relações no sistema solo com o tempo de cultivo no SPD. Desse conjunto devem fazer parte a saturação por $\mathrm{Al}$ e por bases e o $\mathrm{pH}$, com valores de referência de $<5 \%$ para a saturação por $\mathrm{Al}$, de 5,5 para o $\mathrm{pH}$ em água do solo e de $65 \%$ de saturação por bases.

\section{LITERATURA CITADA}

ANGHINONI, I. \& NICOLODI, M. Estratégias de calagem no sistema plantio direto. In: REUNIÃO BRASILEIRA DE FERTILIDADE DO SOLO E NUTRIÇÃO DE PLANTAS, FERTBIO, 27., Lages, 2004. Anais. Lages, Sociedade Brasileira de Ciência do Solo, 2004. CD-ROM
ANGHINONI, I. \& SALET, R.L. Amostragem de solo e as recomendações de adubação e calagem para o sistema plantio direto. In: NUERNBERG, N.J. Conceitos e fundamentos do sistema plantio direto. Lages, Sociedade Brasileira de Ciência do Solo - Núcleo Regional Sul, 1998. p.27-52.

ANGHINONI, I. \& SALET, R.L. Reaplicação de calcário no sistema plantio direto consolidado. In: KAMINSKI, J. Uso de corretivos de acidez do solo no sistema plantio direto. Pelotas, Sociedade Brasileira de Ciência do Solo - Núcleo Regional Sul, 2000. p.41-59.

CAIRES, E.F.; CHUERI, W.Q.; MADRUSA, E.F. \& FIGUEREDO, A. Alterações de características químicas do solo e resposta da soja ao calcário e gesso aplicados na superfície em sistema de cultivos sem preparo do solo. R. Bras. Ci. Solo, 22:27-34, 1998.

CAIRES, E.F.; BANZATO, D.A. \& FONSECA, A.F. Calagem em superfície em sistema plantio direto. R. Bras. Ci. Solo, 24:161-169, 2000.

COMISSÃO DE FERTILIDADE DO SOLO - CFS RS/SC. Recomendações de adubação e de calagem para o Estado do Rio Grande do Sul e Santa Catarina. 3.ed. Passo Fundo, Sociedade Brasileira de Ciência do Solo - Núcleo Regional Sul, Embrapa/CNPT, 1995. 224p.

COMISSÃO DE PESQUISA DE CEVADA. Indicações técnicas para produção de cevada cervejeira: Safras 2001 e 2002. Passo Fundo, Embrapa/CNPT, 2001. 80p.

COMISSÃO DE QUÍMICA E FERTILIDADE DO SOLO - CQFS RS/SC. Recomendações de adubação e calagem para os Estados do Rio Grande do Sul e de Santa Catarina. 10.ed. Porto Alegre, Sociedade Brasileira de Ciência do Solo Núcleo Regional Sul, 2004. 400p.

CONCEIÇÃO, P.C. Avaliação de sistemas de manejo visando à avaliação da qualidade do solo. Santa Maria, Universidade Federal de Santa Maria, 2002. 125p. (Tese de Mestrado)

ESCOSTEGUY, P.A. \& BISSANI, C.A. Estimativa de $\mathrm{H}+\mathrm{Al}$ pelo pH SMP em solos do Rio Grande do Sul e Santa Catarina. R. Bras. Ci. Solo, 23:175-179, 1999.

FIORIN, J.E.; CANAL, I.N.C.; CAMPOS, B.C. \& PETRERE, C.. Fertilidade do solo. In: SILVA, M.T.B., org. A soja em rotação de culturas no sistema plantio direto. Cruz Alta, FUNDACEP/FECOTRIGO, 1998. p.35-96.

GIANELLO, C. \& NICOLODI, M. Relações entre o pH do solo e o rendimento de grãos nos sistemas de cultivos. In: REUNIÃO SUL-BRASILEIRA DE CIÊNCIA DO SOLO, 5., Florianópolis, 2004. Anais. Florianópolis, Sociedade Brasileira de Ciência do Solo - Núcleo Regional Sul, 2004. CD-ROM

KAMINSKI, J. Acidez do solo e a fisiologia das plantas. In: KAMINSKI, J.; VOLKWEISS, J. \& BECKER, F.C. SEMINÁRIO SOBRE CORRETIVOS DA ACIDEZ DO SOLO, 2., Santa Maria, 1989. Seminário... Santa Maria, Universidade Federal de Santa Maria, 1989. p.39-61. 
KAMINSKI, J.; RHEINHEIMER, D.S.; BARTZ, H.R.; GATIBONI, L.C.; BISSANI, C.A. \& ESCOSTEGUY, P.A.V. Proposta de nova equação para determinação do valor de $\mathrm{H}+\mathrm{Al}$ pelo uso do índice SMP em solos do RS e de SC. In: REUNIÃO ANUAL DA REDE OFICIAL DE LABORATÓRIOS DE ANÁLISE DE SOLO E DE TECIDO VEGETAL DOS ESTADOS DO RIO GRANDE DO SUL E DE SANTA CATARINA, 33., Frederico Westphalen, 2001. Ata. Frederico Westphalen, Sociedade Brasileira de Ciência do Solo - Núcleo Regional Sul - ROLAS, 2001. p.21-26.

LOPES, A.S.; WIETHÖLTER, S.; GUILHERME, L.R.G. \& SILVA, C.A. Sistema plantio direto: Bases para o manejo da fertilidade do solo. São Paulo, ANDA, 2004. 110p.

MIELNICZUK, J.; RHEINHEIMER, D.S. \& VEZZANI, F.M. Interações fertilidade e conservação do solo. In: REUNIÃO BRASILEIRA DE FERTILIDADE DO SOLO E NUTRIÇÃO DE PLANTAS, FERTBIO, 25., Santa Maria, 2000. Anais. Santa Maria, Sociedade Brasileira de Ciência do Solo, 2000. CD-ROM

NICOLODI, M.; ANGHINONI, I. \& GIANELLO, C. Relações entre o $\mathrm{pH}$ do solo e os indicadores de fertilidade e o rendimento de soja em lavouras no sistema plantio direto. In: REUNIÃO SUL-BRASILEIRA DE CIÊNCIA DO SOLO, 5., Florianópolis, 2004. Anais. Florianópolis, Sociedade Brasileira de Ciência do Solo - Núcleo Regional Sul, 2004a. CD-ROM

NICOLODI, M.; GIANELLO, C. \& ANGHINONI, I. Fertilidade uma propriedade emergente do sistema solo. In: REUNIÃO SUL-BRASILEIRA DE CIÊNCIA DO SOLO, 5., Florianópolis, 2004. Anais. Florianópolis, Sociedade Brasileira de Ciência do Solo - Núcleo Regional Sul, 2004b. CD-ROM

NOLLA, A. Critérios para a calagem no sistema plantio direto. Porto Alegre, Universidade Federal do Rio Grande do Sul, 2003. 168p. (Tese de Doutorado)

PÖTTKER, D. \& BEN, J.R. Correção da acidez do solo em lavouras sob plantio direto e campo nativo. In: KAMINSKI, J., ed. Uso de corretivos da acidez do solo no plantio direto. Pelotas, Sociedade Brasileira de Ciência do Solo - Núcleo Regional Sul, 2000. p.77-94.

PÖTTKER. D. \& BEN, J.R. Calagem em solos sob plantio direto e em campos nativos do Rio Grande do Sul. In: NUERNBERG, N.J. Conceitos e fundamentos do sistema plantio direto. Lages, Sociedade Brasileira de Ciência do Solo - Núcleo Regional Sul, 1998. p.77-92.
RAIJ, B.van \& QUAGGIO, J.A. Methods used for diagnosis and correction of soil acidity in Brazil: An overview. In: MONIZ, A.C.; FURLANI, A.M.C. \& SCHAFERT, R.E. Plant-Soil Interactions at low $\mathrm{pH}$ : Sustainable agriculture and forestry production. Campinas, Brazilian Soil Science Society, 1997. p.205-214.

RAIJ, B.van; CANTARELLA, H.; QUAGGIO, J.A. \& FURLANI, A.M.C. Recomendações de adubação e calagem no Estado de São Paulo. 2.ed. Campinas, Instituto Agronômico, 1996. 300p. (Boletim Técnico, 100)

REUNIÃO DE PESQUISA DE SOJA DA REGIÃO SUL, 29. Indicações técnicas para a cultura da soja no Rio Grande do Sul e em Santa Catarina 2001/2002. Porto Alegre, FEPAGRO, 2001. 138p.

RIBEIRO, A.C.; GUIMARÃES, P.T.G. \& ALVAREZ V., V.H. Recomendação para o uso de corretivos e fertilizantes em Minas Gerais. Viçosa, MG, CFSEMG/UFV, 1999. 359p.

SÁ J.C. Manejo da fertilidade do solo no sistema plantio direto. In: SIQUEIRA J.O. \& MOREIRA, F.M.S., eds. Interrelação fertilidade, biologia do solo e nutrição de plantas. Lavras, Sociedade Brasileira de Ciência do Solo, 1999. p.267-319.

SALET, R.L. Toxidez de alumínio no sistema plantio direto. Porto Alegre, Universidade Federal do Rio Grande do Sul, 1998. 117p. (Tese de Doutorado)

SILVA, F.C. Manual de análises químicas de solos, plantas e fertilizantes. Brasília, Embrapa Solos, Embrapa Informática Agropecuária, Embrapa Comunicação para Transferência de Tecnologia, 1999. 370p.

SOUSA, D.M.G. \& LOBATO, E. Cerrado: Correção do solo e adubação. 2.ed. Planaltina, Embrapa Cerrados, 2004. 416p.

SUMNER, M.E. Procedures used for diagnosis and correction of soil acidity: A critical review. In: MONIZ, A.C.; FURLANI, A.M.C. \& SCHAFERT, R.E. Plant-Soil Interactions at low $\mathrm{pH}$ : Sustainable agriculture and forestry production. Campinas, Brazilian Soil Science Society, 1997. p.195-204.

TEDESCO, M.J.; VOLKWEISS, S.J. \& BOHMEN, H. Análise de solo, plantas e outros materiais. 2.ed. Porto Alegre, Universidade Federal do Rio Grande do Sul, 1995. 174p. (Boletim Técnico, 5)

VEZZANI, F.M. Qualidade do sistema solo na produção agrícola. Porto Alegre, Universidade Federal do Rio Grande do Sul, 2001. 184p. (Tese de Doutorado)

WIETHÖLTER, S. Calagem no Brasil. Passo Fundo, EmbrapaCNPT, 2000. 104p. 
